\title{
Adaptive Beamsteering for Search-and-Track Application with Cognitive Radar Network
}

\author{
Ric A. Romero \\ Electrical and Computer Engineering \\ Naval Postgraduate School \\ Monterey, CA, USA \\ rnromero@nps.edu
}

\author{
Nathan A. Goodman \\ Electrical and Computer Engineering \\ University of Arizona \\ Tucson, AZ, USA \\ goodman@ece.arizona.edu
}

\begin{abstract}
In this paper, we introduce the concept of a Cognitive Radar Network (CRN). The goal of the radar platforms in a CRN is to cooperate in illuminating the radar channel in an efficient manner in an effort to search for moving targets. Moreover, when a detection is declared, the CRN should incorporate the tracking requirement into the illumination strategy. That is, the beamsteering strategy must exploit the radar channel uncertainty, which is a function of probabilistic representation of the channel. The radar channel uncertainty is dynamic and as such the CRN's beamsteering strategy should be dynamic. Here, we demonstrate a CRN by utilizing two static radar platforms that form a dynamic integrated search-and-track beamsteering strategy matched to the radar channel uncertainty.
\end{abstract}

Index Terms-Cognitive Radar, Cognitive Radar Network, Search and Track, Matched Illumination, Adaptive Beamsteering

\section{INTRODUCTION}

Traditional radar systems form representations of the propagation channel by measuring or estimating the amplitudes (or energies) associated with resolution cells. As opposed to a traditional system, cognitive radar (CR) depicts the radar channel in a probabilistic manner [1-3]. As such, a smart radar should exploit this probabilistic representation to search a surveillance area for moving targets in an efficient manner, i.e., an efficient use of the radar timeline and transmit power via dynamic optimization of the beamsteering strategy. Clearly, in search-and-track applications, both the search function and tracking of detected targets are important priorities. Thus, a $\mathrm{CR}$ should incorporate tracking function priorities into the beamsteering strategy. In [4], a CR platform of this type is introduced, where one platform is able to measure three target parameters, search for targets, and maintain a threeparameter track of detected targets. The three parameters are two position parameters and radial velocity (via a Doppler frequency). When a four-parameter track is desired, then both velocity dimensions are considered. In this paper, it is our interest to demonstrate efficient adaptive beamsteering and platform cooperation between the radars in the network. The objective of the network is to search for targets and establish a four-parameter track of detected targets. Here, we propose to form a cognitive radar network (CRN) with two static radar platforms. The channel's resolution cells are represented such that each cell contains a probability of a target being absent or

\section{CRN FOR FOR SEARCH-AND-TRACK}

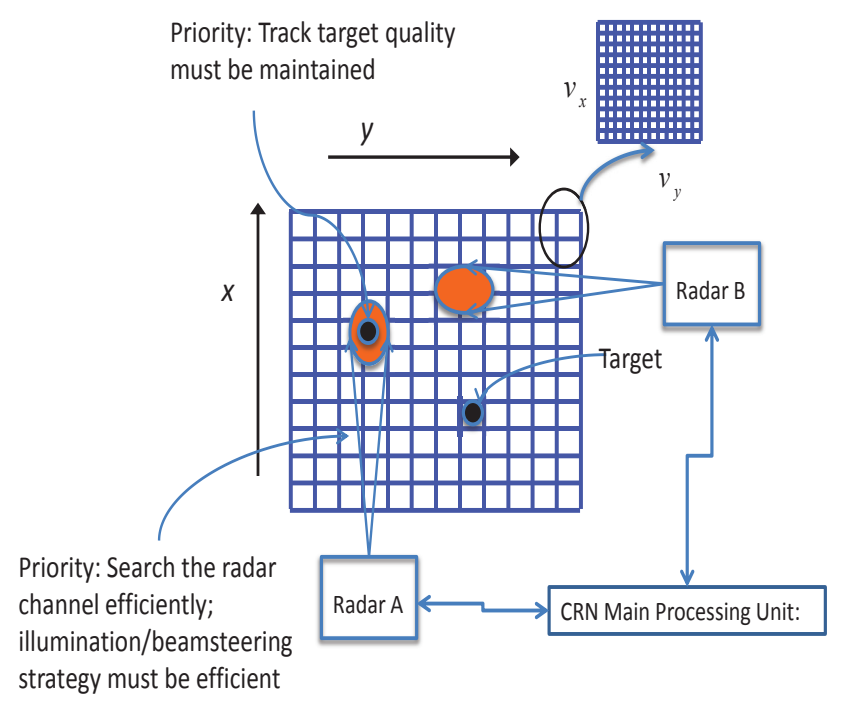

Fig. 1. Overall picture of a CRN for search-and-track application

present. This is called the probabilistic representation of the radar channel. The two radars (each of which can only estimate three parameters as mentioned above) will cooperate such that a four-dimensional (4-D) probabilistic representation can be formed. Moreover, the networked system will form an adaptive beamsteering scheme such that the radar channel uncertainty is reduced thereby yielding an efficient illumination strategy.

\section{Probabilistic Representation and Uncertainty OF THE RADAR CHANNEL}

\section{A. Probabilistic Representation of the Radar Channel}

Fig. 1 shows the surveillance area of interest. For each cell position $(x, y)$, there are various velocity $\left(v_{x}, v_{y}\right)$ possibilities. In radar, target signals correspond to frequencies. Here, we will use antenna and temporal measurements such that physical parameters $\left(x, v_{x}, y, v_{y}\right)$ map to radar-observable parameters $\left(k_{x}, d_{x}, k_{y}, d_{y}\right)$, where $k_{x}$ and $k_{y}$ are spatial frequencies and $d_{x}$ and $d_{y}$ are Doppler frequencies. As such, we have a 4-D 


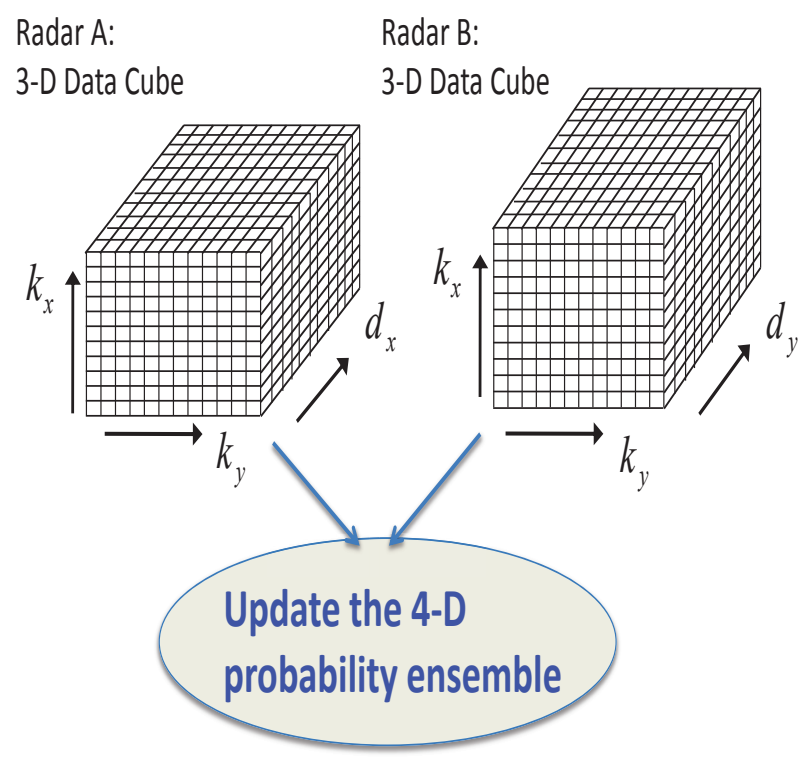

Fig. 2. 3-D measurement cubes used in updating the 4-D probability map

target parameter space in which each resolution cell contains a probability of target present and/or absent. From the geometry above, we note that Radar A is blind to the motion in the $y$ direction; thus resolution cells across $d_{y}$ are ambiguous with respect to Radar A. Similarly, Radar B is blind to $d_{x}$, and thus the probability cells corresponding to this Doppler frequency are ambiguous with respect to Radar B. Clearly, we need a method to update the probability of target presence for all cells due to measurements received from both Radar A and Radar B. The probability updates are dependent on the target model assumptions and the current received measurements, where each radar forms a 3-D measurement cube. This difficult problem is illustrated in Fig. 2. In [3], signal model considerations and probability update methodologies were developed for a twoparameter radar channel that can be extended to our present 4-D scenario. We refer the interested reader to [3].

\section{QUANTIFYING RADAR CHANNEL UNCERTAINTY}

We would like to quantify the radar channel uncertainty in terms of the probabilistic representation. A natural measure of uncertainty of a discrete random variable is entropy, which is given by [5]

$$
h=\sum_{x} p(x) \frac{1}{\ln p(x)},
$$

and is clearly a function of the discrete random variable's probability mass function $p(x)$. We can represent the target presence or absence in a resolution cell as a binary random variable; therefore the uncertainty via (1) is quantified by a cell entropy given by

$$
h_{c}=-p \log _{2} p-(1-p) \log _{2}(1-p),
$$

where $p$ is the probability that a target is in that cell. Fig. 3 shows the cell entropy as a function of the probability that

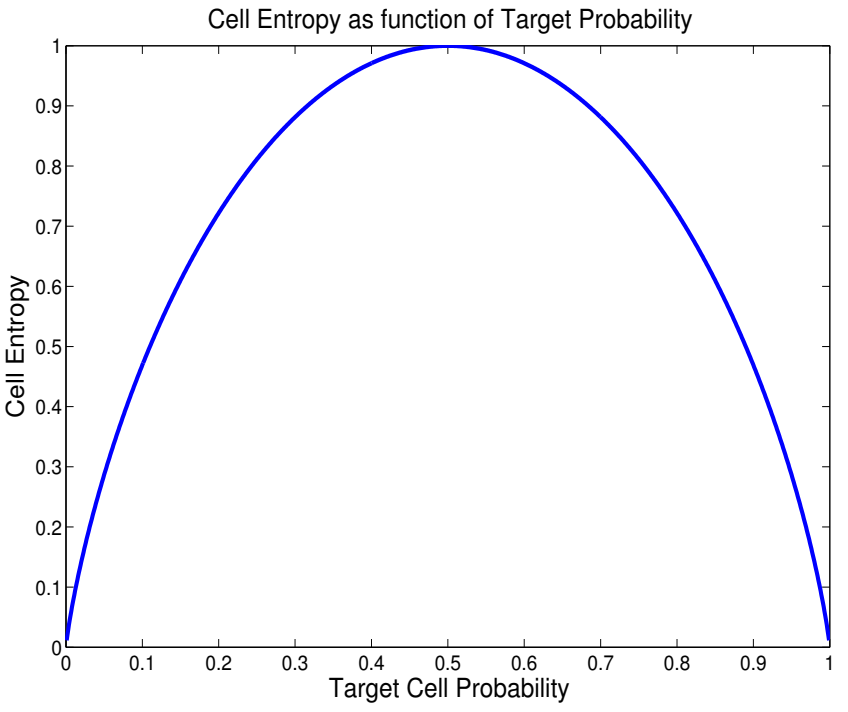

Fig. 3. Overall picture of a CRN for search-and-track application

a target is present (which is exactly the entropy of a binary random variable). Notice that a probability of 0.5 corresponds to the highest cell entropy value of 1 , i.e., 0.5 probability value corresponds to maximum uncertainty. In terms of our forming a beamsteering strategy, areas of high entropies are candidates for immediate illumination.

Now, we can calculate each cell's entropy based on the current probability map. Since we have a 4-D probability map, we have a 4-D entropy map. We propose to use the 4-D entropy map to optimize beam placement for the next illumination, but the illumination is a 2-D function of $\left(k_{x}, k_{y}\right)$. To overcome this issue, we collapse the 4-D entropy map into a 2-D $h_{c}\left(k_{x}, k_{y}\right)$ map by averaging over the Doppler frequencies. Let the area illuminated by the main beamwidth contain $C$ spatial resolution cells. We can calculate the cumulative entropy of any beam position, called beam position entropy (BPE) $h_{P}$, by summing up the individual cell entropies. The BPE is a function of beam position that quantifies the relative importance of interrogating different areas of the scene, at least with respect to the search function. After an illumination of a specific beam position, the target probabilities for all illuminated resolution cells within that particular beam position are updated; thereby producing an updated 4-D entropy map. As stated earlier, a collapsed 2-D entropy map is formed, from which the current BPE map is easily calculated.

\section{Dynamic Probability Model and Kalman TRACKING}

\section{A. Dynamic Probability Model}

Fig. 4 represents the physical surface under surveillance by the CRN. While a beam position is being illuminated by the main beam, targets may appear in un-illuminated beam positions such that the cell probabilities in those areas should be dynamic. In other words, the uncertainty of a target being 


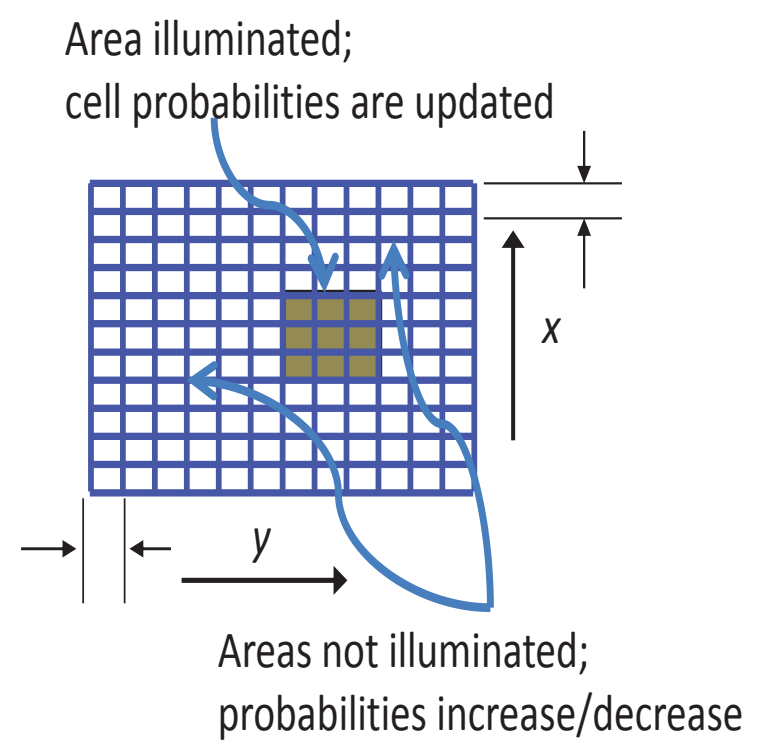

Fig. 4. Dynamic probability model for illuminated and non-illuminated cells

present (or absent) in an un-illuminated cell should adjust to a value that is reflective of some steady-state probability of the cell. We define the steady-state probability of a cell as that probability that is approached in time when a cell is not being updated or illuminated. Here, the probability increase/decrease toward a steady-state probability is implemented as a dynamic model.

In this paper, we will assume all cells to have the same steady-state probabilities. However, in our scenario, we assume that targets are more likely to enter the scene through the boundaries of the scene rather than starting up in the interior of the scene. Referring to Fig. 5, the concentric circles indicate that the outside probabilities converge more rapidly to their steady-state probabilities, which reflects the fact that the scene is more dynamic near its boundaries. That is, when the cells are not being illuminated, the probability rate of increase/decrease of an outside cell is greater than that of an inside cell.

\section{B. Tracking with Kalman Filter}

In addition to searching a scene for new targets, we also desire to track targets that have been detected. For simplicity, in this paper, we utilize the Kalman tracker. In Kalman theory, the state vector is modeled as a Gaussian vector and the tightness of the state vector is given by $\mathrm{K}$, the state covariance matrix (SCM). The uncertainty is quantified by the entropy

$$
h_{t}=0.5 \ln (2 \pi e)^{F} \operatorname{det}(\mathrm{K}),
$$

where $F$ is the length of the state vector. Clearly, $F=4$ since we are interested in a four-parameter track. We refer to (3) as tracked target entropy (TTE). Note that TTE is a linear function of the determinant of the SCM K. The TTE plays an important role in the formation of a search-and-track strategy (as we shall see in the next section). For $M$ detected targets, there are $M$ different TTEs, each quantifying the uncertainty

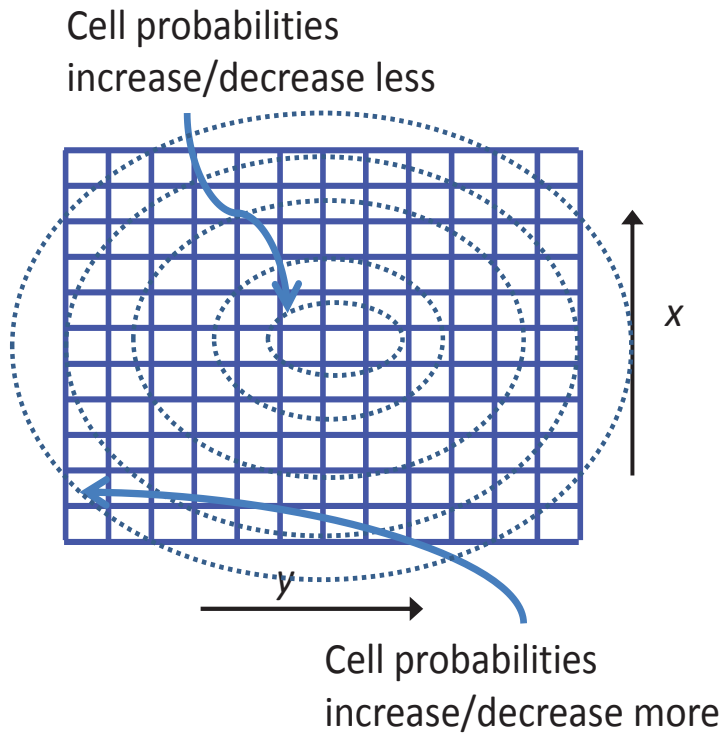

Fig. 5. In this probability change model, large concentric circles indicate higher rates of convergence towards steady-state values

of a particular track. The search-area entropy map (the BPE map) and the track entropies (TTEs) combine to quantify the overall radar channel uncertainty.

\section{Adaptive Search-AND-Track Beamsteering STRATEGY}

Ignoring the track function and concentrating on the search function for the moment, we recognize the way to minimize the post-measurement search-area entropy is to place the beam to the position of highest BPE. This what we call the adaptive search-only beamsteering strategy.

We can incorporate tracking metrics into the beamsteering strategy by a having a desired track entropy threshold, $h_{t, \text { desired. }}$ Once a target is detected, we monitor its TTE. We can integrate track schedule into our adaptive search-only beamsteering strategy in a couple of ways. In the first method, once the threshold $h_{t, \text { desired }}$ is exceeded, we can interrupt the search-only beamsteering strategy and place the beam on to the target. However, a better method is to compare track uncertainties with search uncertainties, i.e., compare BPEs with TTEs. Hence, a more dynamic method of beam position selection is formulated. To accomplish the comparison, we introduce a scalar $\Omega$ such that the two entropies of different types (BPE and TTE) can be compared. For convenience, consider a single detected target. Then the adaptive searchand-track beamsteering strategy rule is then given by

$$
\begin{gathered}
\text { if } h_{t}>\frac{h_{P, \max }}{\Omega} \text { then illuminate target } \\
\text { else illuminate area with max BPE, }
\end{gathered}
$$

where $h_{P, \max }$ is the maximum BPE over all possible beam positions. The scalar $\Omega$ is, therefore, a dial that allows to trade/compromise between search and track priorities. 

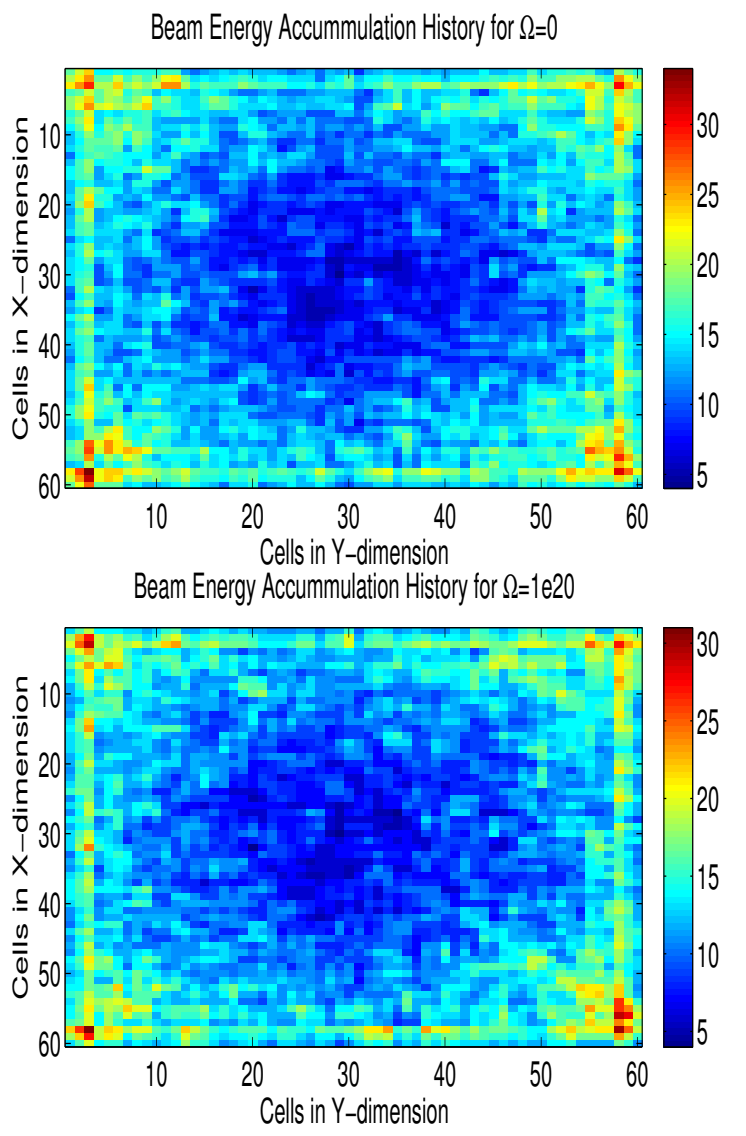

Beam Energy Accummulation History for $\Omega=5 e 20$

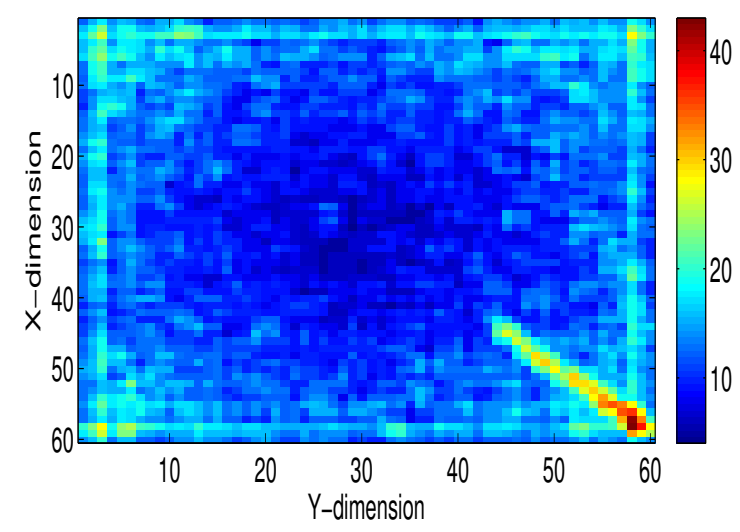

Fig. 6. Beam accumulation histories for different values of $\Omega$. Top panel: $\Omega=0$. Middle panel: $\Omega=1 \mathrm{e} 20$. Bottom panel: $\Omega=5 \mathrm{e} 20$

\section{Simulation Results}

Our goal in this experiment is to use the adaptive searchand-track beamsteering strategy in a search-and-track application. We consider a 4-D scenario with 60-by-60 spatial cells and 15 -by-15 velocity cells per spatial cell. Thus, the overall target parameter space is 60 -by-60-by-15-by-15. We consider three experiments with varying track priority requirements given by: a) $\Omega=0$ represents no track priority requirement, b) $\Omega=1$ e20 represents a slight track priority increase, and c) $\Omega=5$ e20 being the highest track priority requirement of the three. In each experiment, the target initiates motion from the northwest corner to the southeast corner of the scene. Here we will show the results of the experiments in terms of beam accumulation history comparison. Beam accumulation history is simply a graph/metric that shows the number of illuminations spent on each cell of the surveillance area in the course of an experiment. Also, we will show results in terms of track quality comparison as the track priority is increased using the integrated search-and-track beamsteering scheme.

\section{A. Beam Accumulation History Comparison}

The beam accumulation histories for the three experiments are shown Fig. 6. The sub-figures correspond to increasing level of track function priorities: $\Omega=0, \Omega=1 \mathrm{e} 20$, and $\Omega=5 \mathrm{e} 20$. An experiment was generated where a target moved from the left upper corner to the right lower corner. Each radar (alternately) illuminated, collected measurements, and updated the 4-D probability ensemble from the moment the target appeared until it left the scene using the adaptive search-and-track beamsteering strategy. In each case, the target was detected and tracked. The beam accumulation history corresponding to $\Omega=0$ (top panel) reflects the CRN's attempt to match the search-area uncertainty with no regard to the target track. The higher density around the perimeter reflects our dynamic model that implements a bias toward new targets appearing at edges of the search scene. The beam accumulation history corresponding to $\Omega=1 \mathrm{e} 20$ in Fig. 6 (middle panel) reflects the CRN's attempt to put some emphasis on the target track. Notice the slight change in beam accumulation densities from $\Omega=0$ to $\Omega=1 \mathrm{e} 20$. There is an increase in the accumulation density in the southeast corner of the scene, where the target was detected and tracked. The bottom panel corresponds to the beam accumulation history employing $\Omega=5 \mathrm{e} 20$. Here, the pronounced streak near the southeast corner of the scene clearly indicates that target was detected and tracked between the origin and the southeast corner of the scene and that the track priority emphasis is significant.

\section{B. Track Quality Comparison}

We now show the corresponding track position estimates after target detection for the above experiment, where $\Omega=0$, $\Omega=1 \mathrm{e} 20$, and $\Omega=5 \mathrm{e} 20$ correspond to the same experiments above. Fig. 7 shows the positional tracks for the three sample experiments. The left panel shows tracks for the $k_{x}$ position and the right panel shows tracks for the $k_{y}$ position. The target was detected between the origin and the southeast corner of the scene. From the moment of establishing track for this target, there were about 750 time steps (illumination histories) until the target left the scene. Notice the tightness of the track as $\Omega$ is increased. The tightness of the track corresponding to $\Omega=$ $5 \mathrm{e} 20$ is the best (among the three cases) which corresponds to the fact that the illumination update rate for this $\Omega$ is the highest. 

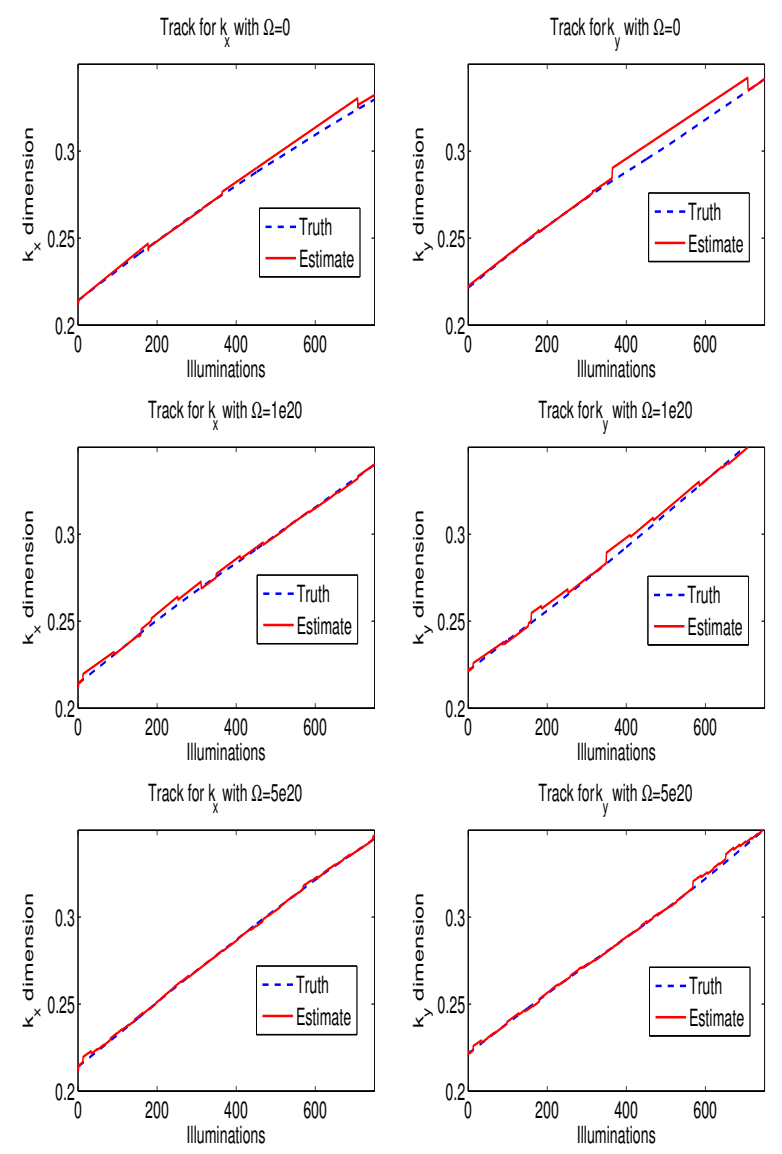

Fig. 7. Positional tracks $\left(k_{x}, k_{y}\right)$ for Scenario Size B (60-by-15-by-60-by15 ) for different values of $\Omega$. Top panel: $\Omega=0$. Middle panel: $\Omega=1 \mathrm{e} 20$. Bottom panel: $\Omega=5 \mathrm{e} 20$

\section{SUMMARY}

In this paper, we introduced the new concept of Cognitive Radar Network (CRN), where the radar platforms in the network cooperate in searching a multidimensional radar channel by exploiting the surveillance area uncertainty. In this paper, the network was composed to two static radar platforms. The channel was represented probabilistically. The appearance of targets and convergence to steady-state probabilities may depend on the actual physical features of the scene. As such a dynamic probability model was introduced. The 4-D probabilistic representation was transformed into search-area entropy map via the beam position entropy (BPE) map. The adaptive search-only beamsteering strategy rule is simply to place the beam to the position with maximum BPE. Track priorities were incorporated by calculating target tracked entropies (TTE). A dynamic illumination scheme (called adaptive search-and-track beamsteering strategy) was formed by introducing a scalar $\Omega$ that allowed comparison of two different entropy types. We presented a series of experiments involving the detection and tracking of a moving target. The experiments corresponded to increasing track priorities. We compared the corresponding beam illumination histories and showed how track priorities and search priorities were accommodated simultaneously. We also showed the corresponding positional tracks for increasing values of $\Omega$. As expected, the tightness of the track improved as the $\Omega$ value was increased. This is because the higher $\Omega$ value corresponded to higher track priority. The higher track priority resulted in a higher illumination update rate, which resulted in a tighter track.

\section{ACKNOWLEDGMENT}

The authors acknowledge support from the ONR via grant \#N000140910338.

\section{REFERENCES}

[1] S. Haykin, "Cognitive radar: A way of the future," IEEE Signal Process. Mag., vol. 23, no. 1, pp. 30-40, Jan. 2006.

[2] N. Goodman, P. Venkata, and M. Neifeld, "Adaptive Waveform Design and Sequential Hypothesis Testing for Target Recognition with Active Sensors, IEEE J. Sel. Topics in Sig. Proc. Mag., vol. 1, no. 1, pp. 105113, Jun. 2007.

[3] R. Romero and N. Goodman, "Channel probability ensemble update for multiplatform radar systems in Proc. IEEE International Waveform Design and Diversity Conf., Niagara Falls, Aug. 2010.

[4] P. Nielsen and N.A. Goodman, "Integrated detection and tracking via closed-loop radar with spatial-domain matched illumination," in Proc. IEEE 2009 Int. Radar Conf., Adelaide, Australia, Sep, 2008

[5] T. Cover and J. Thomas, Elements of Information Theory, John Wiley and Sons, New York, Sep, 1991 